\title{
Statistical Sulcal Shape Comparisons: Application to the Detection of Genetic Encoding of the Central Sulcus Shape
}

\author{
Georges Le Goualher,* Anne Marie Argenti,*† Michel Duyme,† William F. C. Baaré,‡ H. E. Hulshoff Pol,‡ \\ Dorret I. Boomsma,§ Abderrezak Zouaoui," Christian Barillot,\| and Alan C. Evans* \\ *McConnell Brain Imaging Centre, Montréal Neurological Institute, McGill University, Montréal, Quebec H3A 2B4, Canada; †Unité de \\ Recherches d'E pidemiol ogie Génétique, INSERM, U nité 155, U niversité Paris VII, Paris, France; łDepartment of Psychiatry, University \\ Medical Centre Utrecht, Heidel berglaan 100, 3584 CX Utrecht, The Netherlands; §Department of Biological Psychol ogy, Vrije Universiteit, \\ Amsterdam, The Netherlands; "Departement de Neuro-Radiologie, Hopital Sal petriere, Paris, France; and \|French National Research \\ Institute in Computer Science and Control, IRISA, VISTA Project, Rennes, France
}

Received October 7, 1999

Principal Component Analysis allows a quantitative description of shape variability with a restricted number of parameters (or modes) which can be used to quantify the difference between two shapes through the computation of a modal distance. A statistical test can then be applied to this set of measurements in order to detect a statistically significant difference between two groups. We have applied this methodology to highlight evidence of genetic encoding of the shape of neuroanatomical structures. To investigate genetic constraint, we studied if shapes were more similar within 10 pairs of monozygotic twins than within interpairs and compared the results with those obtained from 10 pairs of dizygotic twins. The statistical analysis was performed using a Mantel permutation test. We show, using simulations, that this statistical test applied on modal distances can detect a possible genetic encoding. When applied to real data, this study highlighted genetic constraints on the shape of the central sulcus. We found from 10 pairs of monozygotic twins that the intrapair modal distance of the central sulcus was significantly smaller than the interpair modal distance, for both the left central sulcus $(Z=-2.66 ; P<0.005)$ and the right central sulcus $(Z=-2.26$; $P<0.05)$. Genetic constraints on the definition of the central sulcus shape were confirmed by applying the same experiment to 10 pairs of normal young individuals $(Z=-1.39 ; Z=-0.63$, i.e., values not significant at the $P<0.05$ level) and 10 pairs of dizygotic twins ( $Z=0.47 ; Z=0.03$, i.e., values not significant at the $P<0.05$ level). 02000 Academic Press

Key Words: cerebral cortex; central sulcus; Principal Component Analysis; genetic encoding.

\section{INTRODUCTION}

Cortical sulci of the human brain (and their counterpart gyri) form macroscopic anatomical landmarks on the surface of the brain that are important for spatial localization as well as anatomofunctional correlation studies (Kamada et al., 1993; Watson et al., 1993; Yousry et al., 1997; Dumoulin et al., 1998). Neuroanatomical studies have shown that major sulci occur on every individual brain, indicating a genetic encoding of these entities. In particular, the strict timetable of cortical folding between the 8th and the 44th week of gestation suggests a strong genetic influence on cortical morphometry (Tramo et al., 1995). Since important intersubject cortical variability as well as intrasubject (i.e., interhemispheric) differences have been observed (Ono et al., 1990; Zilles et al., 1997) it is not clear to what extent this variation is a consequence of genetic factors (Tramo et al., 1995).

In the present work, genetic encoding of a neuroanatomical structure was addressed by comparing morphometric statistics from intrapair monozygotic twins with those of interpairs. While dissimilarity between individuals having the same genotype comes unambiguously from environmental factors, similarities can come from genetic factors or environmental factors or both. Therefore, findings obtained from the study of monozygotic twins were confirmed by similar investigations performed on dizygotic twins and unrelated subjects to make an unambiguous conclusion on the influence of genetic factors.

We have previously developed a method (referred to as the Active Ribbon) which models the median surface of a sulcus as a parametric sheet (Le Goualher et al., 1997). Principal Component Analysis (PCA) can then be applied to a collection of such sheets to obtain quantitative information on the variability of sulcal shape (Barillot et al., 1999). This eigenanalysis technique quantifies shape in terms of deformation modes which represent unique and natural coordinates for shape comparisons. In this modal space, modal distance quantifies the observed difference between two shapes. This method allows one therefore to study differences between shapes in a more complete manner than 
studying only differences between subshape characteristics (i.e., length, volume, etc.) as has been done in most previous studies of group differences in morphology. We have applied this statistical shape comparison technique to detect any genetic encoding of the central sulcus shape. The central sulcus is a major fold on the lateral surface of the human brain which separates the sensory from the motor area (White et al., 1997). Previous studies have shown that even though the surface appearance of the central sulcus varies greatly from brain to brain (and between hemispheres of individual brains), its deep structure is remarkably consistent. This apparent contradictory observation leads to a certain confusion in concluding whether the development of this critical feature is strongly controlled by nongenetic or genetic factors.

\section{RELATED WORK}

\section{Studies on Shape Genetic Encoding}

Previous studies have been performed to detect genetic influences on different cerebral structures such as the surface of the corpus callosum (Oppenheim et al., 1979), the asymmetry of the planum temporale (Steinmetz et al., 1995), the global brain volume, and cortical gyrification (Weinberger et al., 1992; Tramo et al., 1995; Steinmetz et al., 1994). These studies have shown that the brain volume and the corpus callosum surface are very similar within pairs of twins. However, studies addressing the genetic encoding of sulcal patterns indicate that the development of the convolutions of the brain is strongly influenced by nongenetic factors (that is, by environment, experience, or chance) (Bonan et al., 1998; Steinmetz et al., 1994). "The important dissimilarities [between twins] can only be understood if we assume that the brain has a particularly high degree of 'developmental freedom'[. . .]The brain is the most individualistic of all organs" (Rössle et al., 1937, cited in Bailey and Von Bonin, 1951). Other observations made of the central sulcus have shown that even if its superficial appearance varies greatly from brain to brain (and from one hemisphere to the other) (Bailey and Von Bonin, 1951; Ono et al., 1990), its deep structure is remarkably consistent. In particular, several authors have noted a strong correlation between the motor hand area and a knob on the precentral gyrus (Yousry et al., 1997; White et al., 1997). This last observation suggests a strong genetic encoding of this particular landmark. In vivo investigation performed by Tramo et al. (1995) on 10 pairs of monozygotic twins has shown that cortical surface area measurements vary more across unrelated twin pairs than within co-twins, suggesting that both the total area and the folding of the cortical surface are heavily influenced by genetic factors in humans.
A weakness of these previous investigations is that they are only qualitative and/or focus on only partial shape characteristics (for example the comparisons of the lengths of the sulci as done in Bonan et al. (1998) or the surface area measurements (Tramo et al., 1995)). We extended these approaches by using a statistical shape comparison technique which allow us to address in a more complete manner the genetic encoding of the central sulcus shape.

\section{Shape Description}

When a numerical representation of a shape (composed generally of a set of feature point coordinates) can be retrieved, methods can be applied to describe not only partial shape characteristics (such as the length, depth, etc.) but overall shape features with a restricted set of parameters. Such shape modeling methods can be distinguished between physical-based modeling methods and statistical modeling.

Using physical modeling of the shapes under study, Sclaroff and Pentland (1995) described a method based on the idea of describing shapes by their generalized symmetries, as defined by the object's vibration or deformation modes. These modes are the eigenvectors of the Finite Element Model of the structure under study and provide an orthogonal, frequency-ordered description of the shape and its natural deformations. Bookstein described described shape deformation in terms of the physical deformation modes of an infinite thin plate (Bookstein, 1989). I nstead of physically modeling the structure under study, researchers have also sought to obtain shape description directly from available samples. In particular, Cootes et al. (1992) PCA to experimentally describe the modes of variation inherent in a training set of 2D heart images. Principal Component Analysis is a statistical technique that finds the directions of maximum variability inherent in a data set and can be applied to different collections of points which could represent curves, surfaces, volumes, or combinations of these possible structures. When applied to 2D outline or 3D surface data, the principal components are called the eigenshapes of the structure under study. Unlike the physical modes, statistical eigenshapes are derived solely from a data set, without the aid of an underlying physical model (only Gaussian distribution for landmark locations is assumed). This can be considered an advantage when one wants to model biological structures for which physical parameters (strength, stress, etc.) are unknown or inappropriate.

Shape modeling methods have been applied to several problems of shape comparisons, in particular in the context of hippocampal morphometry in schizophrenia in which comparisons of neuroanatomical shapes can be more informative than volume comparisons for identifying individuals with neuropsychiatric 
disorders (Csernansky et al., 1998; Martin et al., 1995). Caunce and Taylor (1999) have developed a series of 3D statistical models of the cortical sulci which can be used to locate and label anatomical features automatically in 3D head images. These models allow one to study the main components of the spatial variability of the cortical topography. From our knowledge, none of these shape-modeling methods have been used in the context of sulcal shape comparisons. Considering sulci as freform biological structures (i.e., no particular appropriate physical modeling), and having a method for extracting the median surface of a sulcus, we propose to apply PCA to a collection of such shapes to extract the main components of sulcus shape variability as done in Cootes et al. (1992).

\section{MATERIALS AND METHODS}

\section{D MRI Data}

We had at our disposal 20 tridimensional MRI volumes corresponding to 10 pairs of monozygotic twins (average age 34.6, $\sigma=9.85$; 8 males, 12 females, 19 right handed, 1 left handed). These data were scanned on a General Electric MR scanner GE Signa 1.5 T. Volumes were acquired in sagittal section (SPGR protocol, 124 slices, 1.5-mm interslice distance, $0.9375 \times$ $0.9375-\mathrm{mm}$ intraslice resolution, TR $=23 \mathrm{~ms}, \mathrm{TE}=5$ ms, flip angle $35^{\circ}$ ).

The second group under study is composed of 10 pairs of dizygotic twins (average age 28.5, $\sigma=2.27$; 8 males, 12 females, 19 right handed, 1 left handed) acquired as part of The Netherlands Twin Register program (Boomsma, 1998). These data were scanned on a Philips Gyroscan scanner 1.5 T. Three-dimensional T1-weighted, coronal, spoiled-gradient echo scan (FFE) of the whole head (TE $=4.6 \mathrm{~ms}, \mathrm{TR}=30 \mathrm{~ms}$, flip angle $30^{\circ}$, FOV 256, 170-180 contiguous slices, $1 \times 1 \times$ $1.2-\mathrm{mm}^{3}$ voxels) and a coronal dual-contrast turbo spin echo of the whole brain (TE $1=14 \mathrm{~ms}$, TE2 $=80 \mathrm{~ms}$, $\mathrm{TR}=6350 \mathrm{~ms}$, FOV 256, 120 contiguous slices, $1 \times 1 \times$ $1.6-\mathrm{mm}^{3}$ voxels) were acquired.

We have also included in the following analysis 20 tridimensional MRIs corresponding to 20 subjects selected to match the monozygotic and dizygotic twins' average age, sex, and handedness (average age 29.6, $\sigma=6.22$; 8 males, 12 females; 19 right handed, 1 left handed) from a database of 150 vol unteers corresponding to normal young subjects acquired as part of the International Consortium for Brain Mapping project (Mazziota et al., 1995). These data were acquired on a Philips Gyroscan ACS 1.5-T superconducting magnet system with the following sequence: T1-weighted 3D spoiled-gradient echo acquisition with sagittal volume excitation ( $T R=18, T E=10$, flip angle $30^{\circ}, 140-180$ sagittal slices, slice thickness $1.5 \mathrm{~mm}$ ).

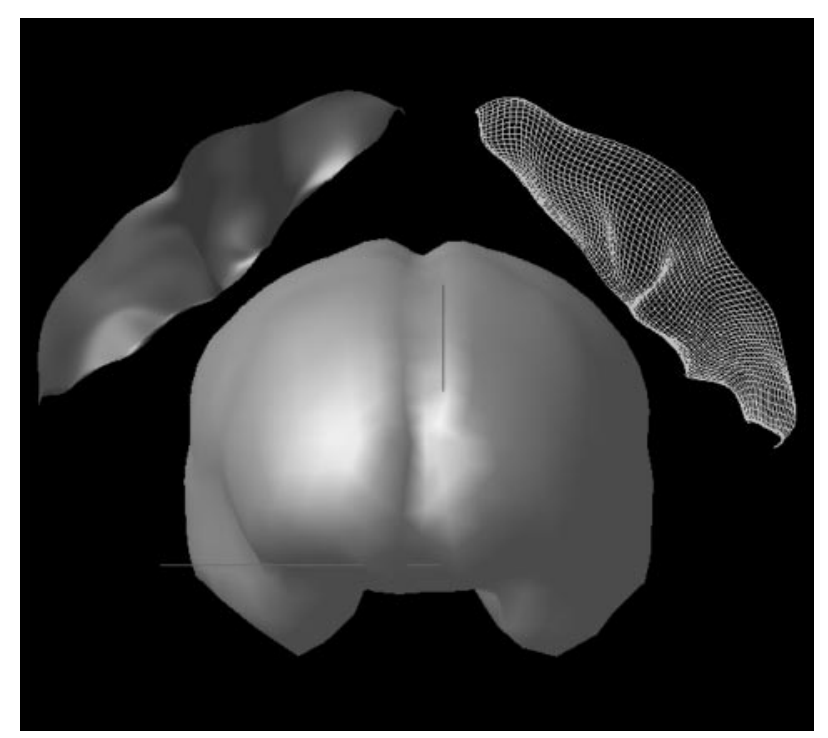

FIG. 1. Median surface of the central sulcus. Result of the modeling of the central sulcus median surface on one particular individual (note that the model brain-displayed at $50 \%$ of its original size-is used only to orient the image).

\section{Cortical Sulcus Modeling U sing Active Ribbons}

We previously developed a method for the 3D segmentation and representation of cortical folds. This method is referred to as the Active Ribbon (Le Goualher et al., 1997). Sulci modeling is obtained from 3D MRI acquisitions. The segmentation is performed using an automatic labeling procedure to separate gyri from sulci based on curvature analysis of the different isointensity surfaces of the 3D MRI volume (Le Goualher et al., 1995). The outer parts of the sulci are automatically detected and used to initialize the convergence of the active ribbon from the exterior opening of the sulcus toward the fundus. This procedure permits the labeling of voxels bel onging to the exterior traces of the sulci as well as on their median surfaces (i.e., buried part of sulcus). Note that it is particularly important to model not only the superficial part of the sulcus, but also its 3D medium surface to perform accurate and complete intersubject shape comparisons. One example of such a modeling approach is presented in Fig. 1.

In the Active Ribbon method the median surface of a sulcus is modeled by a B-spline,

$$
\begin{aligned}
& S(u, v)=(x(u, v), y(u, v), z(u, v)) \\
& =\sum_{j=1}^{n_{c}} \sum_{i=1}^{m_{c}} B_{i, j}(u, v) P_{i, j},
\end{aligned}
$$

where $m_{c}$ is the number of control points along the $u$ parametric direction, and $n_{c}$ is the number of control points along the $v$ direction. Applied to sulcus model- 

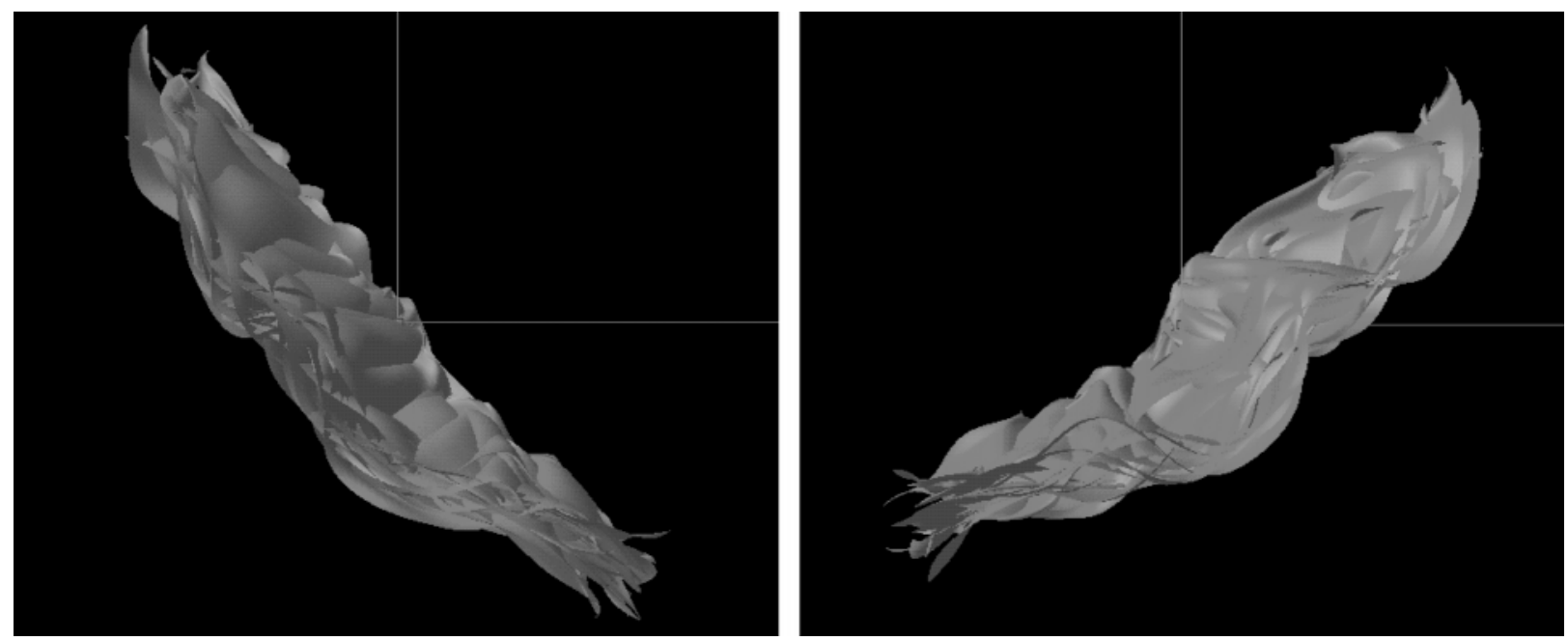

FIG. 2. Residual variability of the central sulcus after Procrustes fit (top view). Left: superposition of the left central sulci extracted on 20 subjects. Right: Superposition of the right central sulci.

ing, $u$ is the parametric index associated with the length of the sulcus and $v$ with its depth. $P_{i, j}$ is a spline control point and $B_{i, j}(u, v)$ is a recursively defined interpolating polynomial of degree 3 (Mortenson, 1985).

The extracted median surface of the central sulcus can then be stored as a vector:

$$
\begin{array}{r}
\vec{S}=\left[x_{1,1}, y_{1,1}, z_{1,1}, \ldots, x_{u, v}, y_{u, v}, z_{u, v}, \ldots,\right. \\
\left.x_{m, n}, y_{m, n}, z_{m, n}\right]^{\top} .
\end{array}
$$

The dimension of such a vector is $3 \times \mathrm{m} \times \mathrm{n}$, where typically $\mathrm{m} \approx 90, \mathrm{n} \approx 30$ ( $\mathrm{m}$ is the total number of sample points along the length of the sulcus, $n$ is the total number of sample points along the depth). The distance between two consecutive sample points is approximately $1 \mathrm{~mm}$. F or a statistical shape comparison, we want to be able to compare the coordinates of equivalent points from different sulcus samples. Therefore, (i) these points must be stored with the same parametric index on each sample so that each point on the mesh can be compared with its counterpart on other surfaces and (ii) all the samples must be aligned in the same manner with respect to a set of axes. The first constraint is achieved by the Active Ribbon process, which uniformly stretches a parametric grid over the entire sulcus median surface. The anatomical correspondence between two different 3D points $S_{k}(u, v)$ and $S_{k^{\prime}}(u, v)$ corresponding to two different subjects $k$ and $k^{\prime}$, stored at the same $(u, v)$ parametrical indices, will be discussed in more detail under Discussion. The second constraint (ii) is obtained by realigning all central sulcus shapes in the minimum variance frame using Procrustes fit (Sibson, 1978; Bookstein, 1991), which finds the translation, rotation, and scale change that allow one to optimally align the ribbons in a sense that the sum of their squared positional differences is minimum. Figure 2 shows the superposition of 20 central sulci after registration. This fitting allows one to remove all spatial differences between sulci and therefore permits one to focus the analysis on shape comparison.

\section{Statistical Shape Modeling U sing PCA}

Each of the previously extracted median surfaces $\vec{S}_{k}$, $\mathrm{k} \in\{1,2, \ldots, \mathrm{N}\}$ is modeled by a $\mathrm{B}$-spline and can therefore be represented by the equation

$$
\vec{S}_{k}=B_{k} \vec{P}_{k}
$$

where $B$ is the bending matrix and $\vec{P}$ is the control points vector (Mortenson, 1985).

The Principal Component Analysis involves the computation of a mean shape. To compute such a mean shape, all parametric meshes should be of the same resolution (i.e., should have the same nodal structure). However, as our parameterization is uniform along both the length and the depth of a sulcus, each ribbon is composed of a number of sampling points, which depends on the actual length and depth of corresponding sulcus and thus varies from one subject to another. Therefore, to end up with a constant number of sample points we find the maximum extent along each parametric direction $\mathrm{u}$ and $\mathrm{v}$ from our set of $\mathrm{N}$ surfaces, say $m_{\text {sup }}$ and $n_{\text {sup }}$, and resample all the extracted surfaces $\vec{S}_{k}$ with this constant number of sample points. We then set the number of control points to $\frac{1}{4}$ the number of points along the two parametric directions: $m_{c}=\frac{1}{4} m_{\text {sup }}$ and $\mathrm{n}_{\mathrm{c}}=\frac{1}{4} \mathrm{n}_{\text {sup }}$. This ratio is referred to as the spline smoothing factor: if $\mathrm{m}_{\mathrm{c}}=\mathrm{m}_{\text {sup }}$ and $\mathrm{n}_{\mathrm{c}}=\mathrm{n}_{\text {sup }}$ the spline is the interpolating spline. The smoothing is all the 

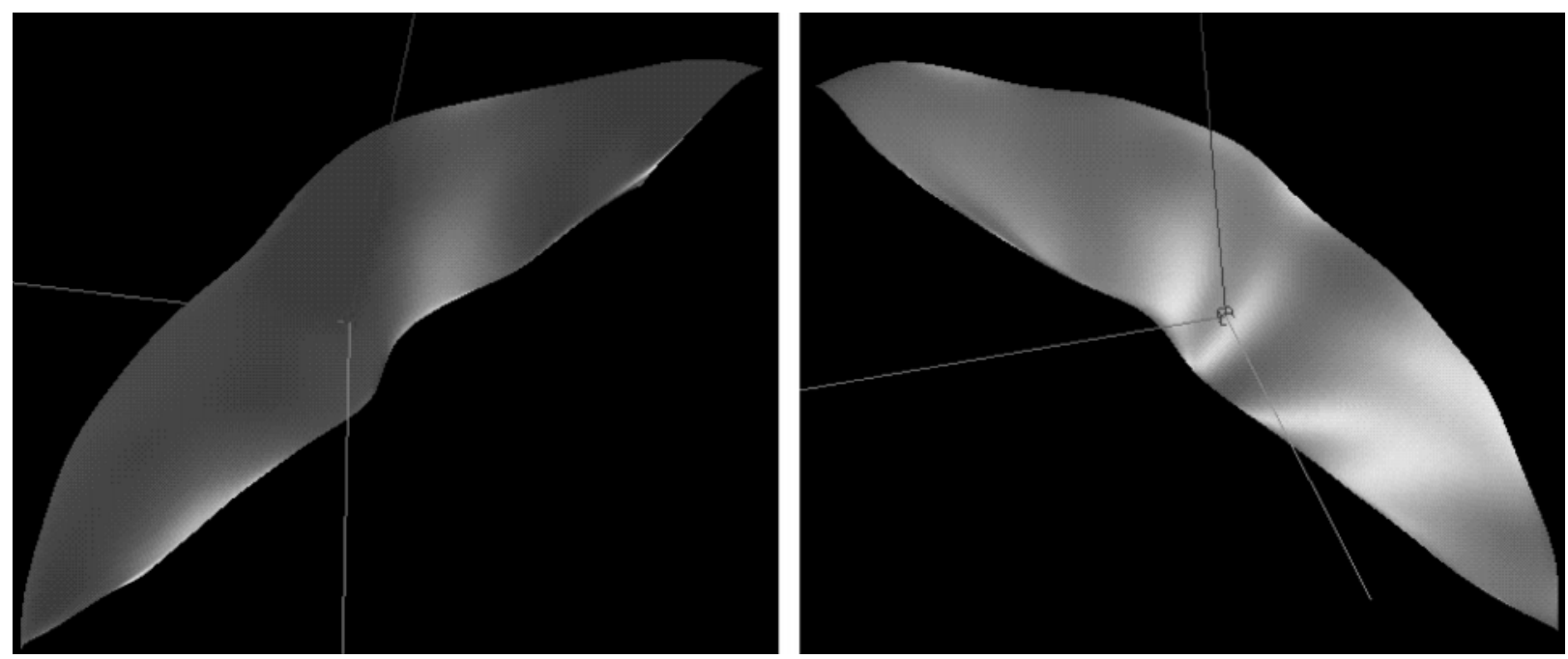

FIG. 3. Shape of the mean central sulcus computed by averaging 20 samples. Left: Right central sulcus. Right: Left central sulcus.

more important as $m_{d} / m_{\text {sup }}$ and $n_{d} / n_{\text {sup }}$ decrease. We found that a value of $\frac{1}{4}$ allows us to obtain a good approximation of the sulcus median surface when using a bicubic spline (Le Goualher et al., 1997). Using this ratio the dimension of vector $\vec{P}$ becomes $3 \times m_{c} \times$ $\mathrm{n}_{\mathrm{c}}$ (16 fewer components in vector $\overrightarrow{\mathrm{P}}$ than in vector $\overrightarrow{\mathrm{S}}$ ). In order to reduce the amount of computation, we take advantage of this spline modeling and apply the PCA to the control points vector $\vec{P}$ rather than the sampling points vector $\overrightarrow{\mathrm{S}}$.

Following resampling, each shape, $\vec{S}_{k}$ is described by a control point vector $\overrightarrow{\mathrm{P}}_{\mathrm{k}}$ of constant dimension $3 \times$ $m_{c} \times n_{c}$, allowing therefore the computation of the mean shape of the central sulcus:

$$
\begin{aligned}
& \overrightarrow{\mathrm{P}}_{\text {mean }}=\frac{1}{\mathrm{~N}} \sum_{\mathrm{k}=1}^{\mathrm{N}} \overrightarrow{\mathrm{P}}_{\mathrm{k}} \text {, } \\
& \overrightarrow{\mathrm{S}}_{\text {mean }}=\mathrm{B} \overrightarrow{\mathrm{P}}_{\text {mean }} .
\end{aligned}
$$

As matrix $B$ is constant (all surfaces resampled with the same number of points), there is an equivalence between Eq. (5) and $\vec{S}_{\text {mean }}=1 / N \sum_{k=1}^{N} \vec{S}_{k}$ (Fig. 3 shows the mean central sulcus). Then to capture shape variability, the global covariance matrix is diagonalized:

$$
\begin{aligned}
& C=\frac{1}{N} \sum_{k=1}^{N}\left(\vec{P}_{k}-\vec{P}_{\text {mean }}\right)\left(\vec{P}_{k}-\vec{P}_{\text {mean }}\right)^{\top}, \\
& C=\Phi \Lambda \Phi^{\top}, \\
& \Lambda=\operatorname{diag}\left(\lambda_{1}, \lambda_{2}, \ldots, \lambda_{3 \times n_{c} \times m_{c}}\right), \quad \text { with } \lambda_{1}>\lambda_{2},
\end{aligned}
$$

with $\lambda_{\mathrm{i}}\left(\mathrm{i}=1, \ldots, 3 \times \mathrm{n}_{\mathrm{c}} \times \mathrm{m}_{\mathrm{c}}\right)$ the eigenvalues of the covariance matrix $C$ and $\Phi$ the matrix containing the eigenvectors. Note that if the training set consists only of $\mathrm{N}<3 \times \mathrm{n}_{\mathrm{c}} \times \mathrm{m}_{\mathrm{c}}$ samples, there will be only $\mathrm{N}-1$ linearly independent columns or rows in $\Phi$ and the same number of eigenmodes in $\Lambda$.

One particular sulcus can then be written:

$$
\begin{gathered}
\overrightarrow{\mathrm{P}}_{\mathrm{k}}=\overrightarrow{\mathrm{P}}_{\text {mean }}+\Phi \overrightarrow{\mathrm{b}}_{\mathrm{k}} \quad \text { with } \overrightarrow{\mathrm{b}}_{\mathrm{k}}=\Phi^{\top}\left(\overrightarrow{\mathrm{P}}_{\mathrm{k}}-\overrightarrow{\mathrm{P}}_{\text {mean }}\right), \\
\overrightarrow{\mathrm{S}}_{\mathrm{k}}=\mathrm{B} \overrightarrow{\mathrm{P}}_{\mathrm{k}} .
\end{gathered}
$$

The vector $\vec{b}_{k}$ contains modal amplitudes representing the difference between sulcus $\vec{S}_{k}$ and the reference shape $\vec{S}_{\text {mean }}$ in the eigenvector space. $\Phi$ contains the eigenvectors and is constant. An approximation $\vec{S}_{k}$ of the observed sample $\vec{S}_{k}$ is obtained by truncating $\Phi$ and $\vec{b}_{k}$ in order to keep only the $\mathrm{t}\left(\mathrm{t} \ll \min \left(\mathrm{N}-1,3 \times \mathrm{n}_{\mathrm{c}} \times \mathrm{m}_{\mathrm{c}}\right)\right.$ ) most significant modes of variations. $t$ is determined using the criterion

$$
\frac{\sum_{\mathrm{i}=1}^{\mathrm{t}} \lambda_{\mathrm{i}}}{\sum_{\mathrm{i}=1}^{3 \times n_{\mathrm{c}} \times \mathrm{m}_{\mathrm{c}}} \lambda_{\mathrm{i}}} \geq \tau,
$$

where $\lambda_{i}$ is the ith eigenvalue of $\Lambda, \lambda_{i} \geq \lambda_{i+1}$, and $\tau$ is a predefined proportion of the total variation (in our experiments we used $\tau=0.98$ ). At a 98\% approximation, for $\mathrm{N}=20$ samples, we found that the root mean square distance between a sample $\vec{S}_{k}$ and its approximation $\overrightarrow{\mathrm{S}}_{\mathrm{k}}=\mathrm{B} \overrightarrow{\mathrm{P}}_{\mathrm{k}}$, with $\overrightarrow{\mathrm{P}}_{\mathrm{k}}=\overrightarrow{\mathrm{P}}_{\text {mean }}+\tilde{\Phi}_{\mathrm{b}}$, was typically only $0.4 \pm 0.1 \mathrm{~mm}$. At this approximation level, the dimension of $\vec{b}$ is $t \approx 15$, meaning that only 15 major deformation modes are needed to quantify the difference between $\vec{S}_{k}$ and $\vec{S}_{\text {mean }}$.

\section{Modal Distance}

In the eigenvector space, the modal distance between two samples is defined as 


$$
\mathrm{d}\left(\mathrm{S}_{\mathrm{i}}, \mathrm{S}_{\mathrm{j}}\right)=\left\|\overrightarrow{\mathrm{b}}_{\mathrm{j}}-\overrightarrow{\mathrm{b}}_{\mathrm{i}}\right\|
$$

The closer this norm is to zero, the more similar the two samples.

\section{Detection of a Genetic Encoding}

If a genetic encoding exists, one can argue that the minimum sulcal shape distance $d_{\min }\left(S_{i}, S_{j}\right)$ will systematically occur within a pair of twins, i.e., $d\left(S_{a}, S_{b}\right) \leq$ $\mathrm{d}\left(\mathrm{S}_{\mathrm{a}}, \mathrm{S}_{\mathrm{j}}\right), \forall \mathrm{S}_{\mathrm{j}}$, if $\mathrm{S}_{\mathrm{a}}$ and $\mathrm{S}_{\mathrm{b}}$ are from monozygotic twins. However, this assumption may not al ways be true even if a genetic encoding exists simply by chance. We need therefore to define a statistical test to show that the intrapair modal distances are statistically smaller than the interpair modal distances.

The statistical test used in this work was the Mantel permutation test (Mantel, 1967). The Mantel permutation test requires no assumptions for population distribution and it makes meaningful use of all available data. We briefly recall this test and for more detailed information refer the reader to Worsley et al. (1991). Consider the $\mathrm{N} \times \mathrm{N}$ symmetrical matrix $\mathrm{R}_{i, j}=\mathrm{d}\left(\mathrm{S}_{i}\right.$, $\left.S_{j}\right)=\left\|\vec{b}_{j}-\vec{b}_{i}\right\|$, which stores the modal distances between shapes $S_{i}$ and $S_{j}$ and the $N \times N$ matrix $D_{i, j}{ }^{1}$ where

$$
\left\{\begin{array}{l}
D_{i, j}=1 \text { if } i \text { and } j \text { are twins } \\
D_{i, j}=0 \text { if not. }
\end{array}\right.
$$

The statistic

$$
F_{0}=\sum_{j=1}^{N} \sum_{i=1}^{N} R_{i, j} D_{i, j}
$$

assesses the modal distances for twin pairs. This statistic is then compared with the distribution of the random variable

$$
F=\sum_{j=1}^{N} \sum_{i=1}^{N} R_{P(i) P(j)} D_{i, j}
$$

where $P(1), P(2), \ldots, P(N)$ are random permutations of the subject indices $1,2, \ldots, N$.

Shapiro and Hubert (1979) proved that, under certain conditions, the standardized statistic

$$
G=\left[F_{0}-E(F)\right] / \operatorname{var}(F)^{1 / 2}
$$

is asymptotically normally distributed for large N. Then if the distribution of the intrapair modal distances is similar to the distribution of the interpair

\footnotetext{
${ }^{1}$ Note that the diagonal of $R$ as well as $D$ is 0 .
}

modal distances (i.e., no genetic encoding), the value of $\mathrm{F}_{0}$ will belong unambiguously to the normal distribution $\mathcal{N}(E(F), \operatorname{var}(F))$. On the contrary, for a "twin effect" on modal distances (i.e., distribution of the intrapair modal distances differs from the distribution of the interpair modal distances) the value obtained for $F_{0}$ will belong with a very low probability to the normal distribution $\mathcal{N}(E(F)$, var $(F))$. Then a statistical difference between intra- and interpair modal distances can be detected by computing the standardized statistic $G$ (cf. Eq. (15)) and comparing this value with a cumulative normal distribution table (as performed for the classical Z test (Crow et al., 1990): for a statistical difference between intra- and interpair modal distances, $\mathrm{G}$ bel ongs with a very low probability to $\mathcal{N}(0)$,$) .$

\section{RESULTS}

\section{Active Ribbon Validation: Comparisons and Automatic and Manual Segmentation}

In this section, we investigated whether sulci modeling performed using the Active Ribbon method produces results comparable with those obtained manually (we also refer the reader to Le Goualher et al. (1999) for a more complete validation study). In order to compare the active ribbon versus the manual labeling, we measured the root mean square distance (noted $\sigma)$ between the two digital representations. The root mean square is computed as

$$
\begin{aligned}
\sigma_{\text {am }}^{2} & =\frac{1}{\mathrm{~N}\left(\Omega_{\text {auto }}\right)} \sum_{\nu \in \Omega_{\text {auto }}}\left(\mathrm{d}^{\mathrm{am}}(\nu)\right)^{2}, \\
\sigma_{\text {ma }}^{2} & =\frac{1}{\mathrm{~N}\left(\Omega_{\text {manual }}\right)} \sum_{\nu \in \Omega_{\text {manual }}}\left(\mathrm{d}^{\mathrm{ma}}(\nu)\right)^{2},
\end{aligned}
$$

with $N\left(\Omega_{\text {manual }}\right)$ as the number of voxels manually labeled and $\mathrm{N}\left(\Omega_{\text {auto }}\right)$ as the corresponding set of voxels labeled with the Active Ribbon method. Distance measures ( $d^{\text {am }}$ and $d^{\text {ma }}$ ) were calculated by computing a distance transform (Borgefors, 1984), which gives for each point of the parameterized surface the distance to its closest point in the manually traced sulcus. We denote $\mathrm{d}^{\text {am }}$ the distance between the automatic and the manual labeling. We also measured the error $\mathrm{d}^{\text {ma }}$ which represents the distance between the manual and the automatic labeling. These two measures are complementary because they allow us to respectively quantify over- and underestimations. For instance, when considering a sulcus which is only partially covered with our Active Ribbon method the first error $\mathrm{d}^{\text {am }}$ will be small, but $d^{\text {ma }}$ will have a high value.

The root mean square was computed between automatic and manual labelings of the central sulcus performed on 10 pairs of monozygotic twins. As can be 


\section{TABLE 1}

Root Mean Square Distances (Denoted $\sigma$ ) between Manual and Active Ribbon Labeling for $\mathrm{N}=20$ Sulci

\begin{tabular}{ccccccc}
\hline & \multicolumn{2}{c}{ Auto vs manual } & & \multicolumn{2}{c}{ Manual vs auto } \\
\cline { 2 - 3 } \cline { 6 - 7 } & Mean $\sigma_{\text {am }}$ & Max $\sigma_{\text {am }}$ & & Mean $\sigma_{\text {ma }}$ & Max $\sigma_{\text {mannnn}}$ \\
\hline SCL & 1.16 & 3.32 & & 0.91 & 2.51 \\
SCR & 1.41 & 2.65 & & 1.70 & 2.66 \\
\hline
\end{tabular}

Note SCL, central sulcus of left hemisphere; SCR, central sulcus right.

seen in Table 1, the mean $\sigma$ distance for manual vs active ribbon labeling has a value of $\sigma_{\mathrm{ma}}=(0.91+$ $1.70) / 2=1.31 \mathrm{~mm}$. The mean value of $\sigma$ when active ribbon labeling was taken as the standard is $\sigma_{\mathrm{am}}=$ $(1.16+1.41) / 2=1.29 \mathrm{~mm}$. A symmetric error estimate is given by the mean value of these two measures and has a value of $\sigma=(1.31+1.29) / 2=1.30 \mathrm{~mm}$. This means that the average error between two labelings is less than 2 voxels for a $1-\mathrm{mm}^{3}$ isotropic MRI volume. These results show that the 3D structure of the cortical sulci as retrieved by the active ribbon modeling is similar to that obtained by manual labeling.

\section{Simulated Data}

To study the ability of PCA associated with the Mantel permutation test to detect differences between intra- and interpair morphometrics of the central sulcus shape, we applied this methodology to simulated data.

Simulation of Homogeneous Samples (i.e., No Genetic Encoding)

The goal of this first experiment was to generate a set of homogeneous shapes and to apply the Mantel permutation test on computed modal distances to verify that $G$ was close to zero (i.e., $G$ belongs with a very high probability to $\mathcal{N}(0,1)$ ) when no "twin effect" was present.

Random spatial deformations were first generated using the following procedure: a set of 20 landmarks was defined within a reference 3D MRI brain. A deformed set of these landmarks was then produced by adding a random displacement to each of the landmark coordinates (since previous work in our laboratory has shown neuroanatomical variability to be on the order of 4-7 mm in 3D (Sorlié et al., 1997), a Gaussian random number generator with standard deviation of $4 \mathrm{~mm}$ was used to produce the individual components of the displacement vector). The two resulting landmark point sets (original and deformed) were then used to define a continuous 3D thin-plate spline (TPS) transformation function (Bookstein, 1989). This interpolant was used to resample the original data and produce a spatially warped source data set for testing.
Twenty 3D deformations were generated using this method and used to deform a reference $S_{\text {ref }}$ sulcal shape into 20 warped versions. The average deformation magnitude was $5 \mathrm{~mm}$ with a maximum of $16 \mathrm{~mm}$. PCA was then applied to this set of 20 shapes and modal distances were computed between each couple $\left(S_{i}, S_{j}\right)$ $(i, j=1, \ldots, 20)$, with $S_{i}$ the warped version of $S_{r e f}$ generated by the ith Gaussian random field $(i=1, \ldots$, 20). These modal distances were stored in the following symmetrical matrix R:

\begin{tabular}{c|ccccc} 
& $\mathrm{S}_{1}$ & $\mathrm{~S}_{2}$ & $\mathrm{~S}_{3}$ & $\cdots$ & $\mathrm{S}_{20}$ \\
\hline $\mathrm{R}=\mathrm{S}_{1}$ & 0 & $\mathrm{~d}_{2}^{\text {intra }}(1,2)$ & $\mathrm{d}^{\text {inter }}(1,3)$ & $\cdots$ & $\mathrm{d}^{\text {inter }}(1,20)$ \\
$\vdots$ & $\mathrm{d}^{\text {intra }}(2,1)$ & 0 & $\mathrm{~d}^{\text {inter }}(2,3)$ & $\cdots$ & $\mathrm{d}^{\text {inter }}(2,20)$ \\
$\mathrm{S}_{20}$ & $\vdots$ & $\vdots$ & $\vdots$ & $\vdots$ & $\vdots$ \\
& $\cdots$ & $\cdots$ & $\cdots$ & $\cdots$ & 0
\end{tabular}

Subject pair $\left\{\left(\mathrm{S}_{1}, \mathrm{~S}_{2}\right),\left(\mathrm{S}_{3}, \mathrm{~S}_{4}\right), \ldots,\left(\mathrm{S}_{19}, \mathrm{~S}_{20}\right)\right\}$ modal distances (denoted $d^{\text {intra }}$ ) were then compared with all other modal distances ( $d^{\text {inter }}$ ) through the computation of G (cf. Eq. (15)). Since from this experiment construction we knew that there was no particular difference between these two groups of measurements (i.e., in this first set of experiments, genetic encoding was not incorporated into the simulation), we expected to find $\mathrm{G} \sim 0$.

Figure 4 shows the distribution of the values of $G$ obtained for 50 experiments (i.e., computation of $G$ for 50 different simulated data sets of 20 sulci). As expected one can see that the $G$ values were cl ose to zero (mean value 0.02; $\sigma=0.88$, range $[-1.7392 ; 2.1165]$ ). In order to conclude the existence of a significant difference between arbitrary pairs $\left\{\left(\mathrm{S}_{1}, \mathrm{~S}_{2}\right),\left(\mathrm{S}_{3}, \mathrm{~S}_{4}\right), \ldots\right.$, $\left(S_{19}, S_{20}\right)$ \}and all other interpair distances, the value of $\mathrm{G}$ must be compared with a cumulative normal distribution table (Crow et al., 1990). In this experiment, G values are close to zero (as expected for this homogeneous set of samples) and thus no significant difference between intrapair and interpair distances was found at $P<0.01$. One simulation in 50 gave a statistical significance $(G=2.1165)$ at the $P<0.05$ level.

In conclusion, the simulations have shown that when comparing $G$ with a cumulative normal distribution table a significant difference between intrapair and interpair distances was, as expected, never found at $\mathrm{P}<0.01$.

\section{Simulation of Pairs of Twins: Genetic Encoding Included}

We generated 10 simulated pairs of twins using two different standard deviations for the random number generator. The first standard deviation was $4 \mathrm{~mm}$ as used previously and was used to generate 10 independent samples $\left(\mathrm{S}_{1}, \mathrm{~S}_{3}, \ldots, \mathrm{S}_{19}\right)$. The second standard deviation had a value of only $2 \mathrm{~mm}$ and was used to 


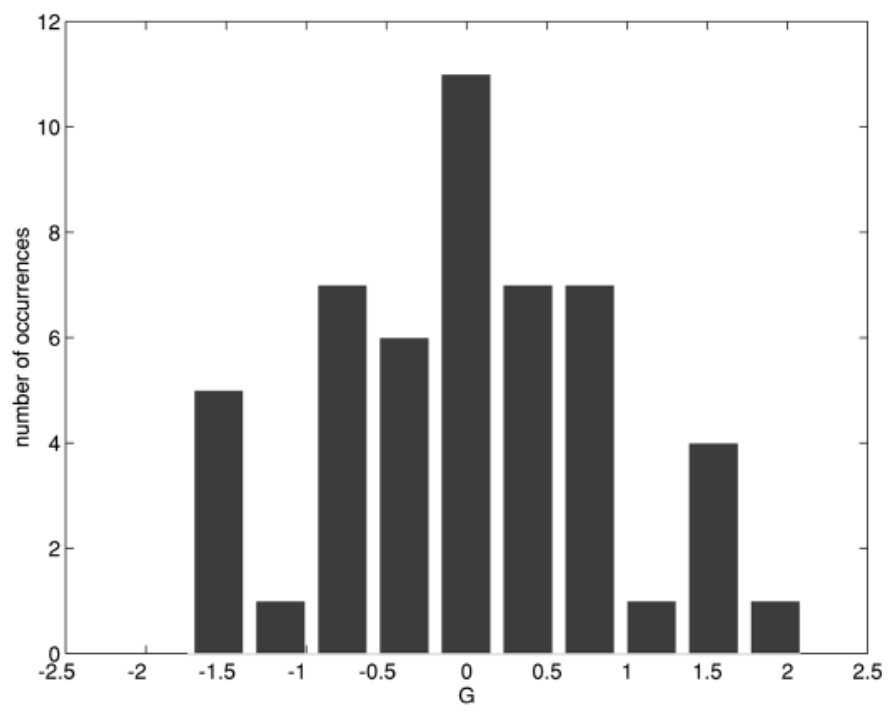

FIG. 4. Statistic of $\mathrm{G}$ obtained from 50 simulations of 20 independent subjects. This statistic is supposed to follow a normal distribution of mean 0 and standard deviation 1. Experimental mean found: 0.0212. Experimental standard deviation: 0.8836 .

generate the simulated twin for each of the computed samples. We denote $\left\{\left(\mathrm{S}_{1}, \mathrm{~S}_{2}\right),\left(\mathrm{S}_{3}, \mathrm{~S}_{4}\right), \ldots,\left(\mathrm{S}_{19}, \mathrm{~S}_{20}\right)\right\}$ the 10 simulated pairs of twins. Note that $S_{2}$ is the deformed version of $\mathrm{S}_{1}$ obtained form a TPS transformation associated with a random number generator of mean zero and $2 \mathrm{~mm}$ standard deviation. The average TPS deformation magnitude between two simulated twins was $2 \mathrm{~mm}$ with a maximum of $6 \mathrm{~mm}$ (remember that the average TPS deformation magnitude between two independent subjects was $5 \mathrm{~mm}$, with a maximum of $16 \mathrm{~mm}$ ).

Fifty simulations were performed as before. The histogram of the $G$ values obtained from these experiments is shown in Fig. 5. The mean value of $\mathrm{G}$ is -7.38 and its standard deviation 0.54 , with a range [-8.55; -6.19]. Upon comparison with a cumulative normal distribution table, the values were found to be statistically significant at the $\mathrm{P}<1.10^{-14}$ level. Therefore, as expected, a significant difference between intrapair and interpair modal distances was found in all the simulations.

\section{Conclusion}

These two sets of simulations have shown the wellsuited behaviour of the Mantel permutation test, applied to modal distances, for detecting any genetic encoding of sulcal shapes.

\section{Statistical Analysis of Modal Distances on Independent Subjects}

The first analysis on real data was performed on a group of 20 young normal subjects obtained as part of an ongoing project to study normal neuroanatomical variation. The group of $\mathrm{N}=20$ subjects was chosen such that it matched the twins group for age, sex, and handedness. Subjects were labeled from 1 to 20 and arbitrary pairs $\left\{\left(\mathrm{S}_{1}, \mathrm{~S}_{2}\right),\left(\mathrm{S}_{3}, \mathrm{~S}_{4}\right), \ldots,\left(\mathrm{S}_{19}, \mathrm{~S}_{20}\right)\right\}$ modal distances were compared with interpair distances. This experiment is similar to our first simulation as we have a group of homogeneous samples in which no "twin effect" is present, and therefore a theoretical value of $G$ close to zero should be retrieved.

Values obtained were $G=-1.39$ for the left central sulcus and $G=-0.63$ for the right hemisphere. None of these values were significant at the $\mathrm{P}<0.05$ level. As expected, no particular stronger similarity was observed for the central sulcus shape in our arbitrary pairs compared to all other possible pairs.

\section{Statistical Analysis of Modal Distances on Monozygotic Twins}

We then computed the modal distances for the central sulci extracted from the database of 10 pairs of monozygotic twins. The $G$ value obtained for the left hemisphere was $G=-2.66$, indicating that the intrapair modal distances were statistically $(P<0.005)$ smaller than the interpair distances. This suggests a genetic encoding of the shape of the left central sulcus.

When concerned with the right hemisphere, we obtained a $G$ value of -2.26 . This value is statistically significant at the $P<0.05$ level. Then for both hemispheres a correlation for central sulcus modal distances appears clearly for monozygotic twins, suggesting a genetic encoding of the shape of this major neuroanatomical landmark. However, as similarities can arise from genetic factors or environmental factors,

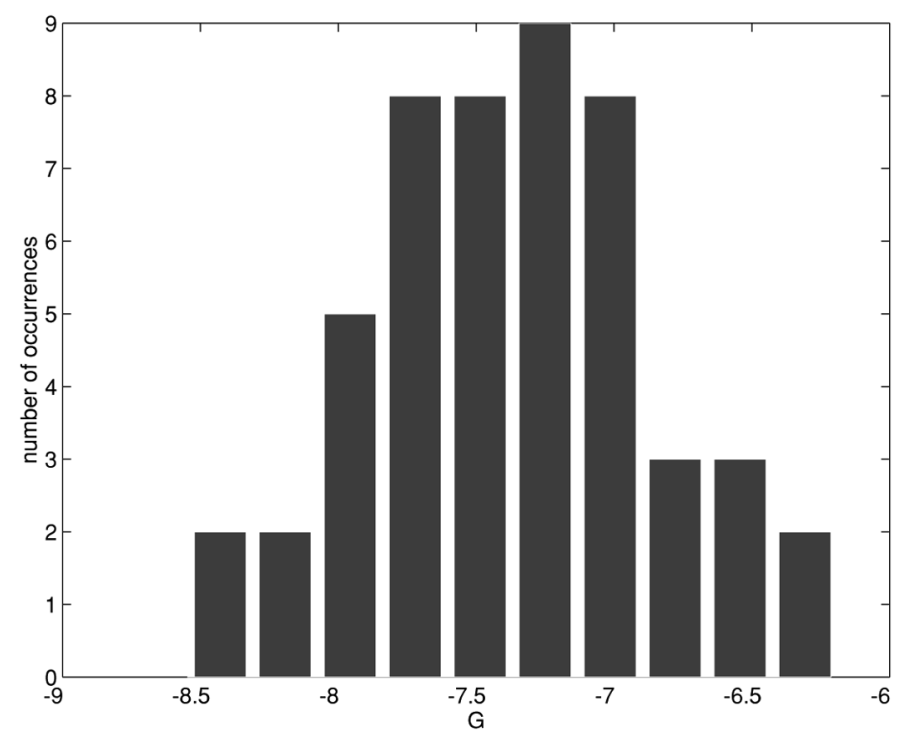

FIG. 5. Statistic of $\mathrm{G}$ for simulated twins obtained form $50 \mathrm{sim}$ ulations. Experimental mean found: -7.3822. Experimental standard deviation: 0.5385 . 
or both, a complementary study had to be done on dizygotic twins.

\section{Statistical Analysis of Modal Distances on Dizygotic Twins}

Adding a group of dizygotic twins to the experiment allows us to study more completely the influence of a genetic factor in the definition of the central sulcus shape. As the dizygotic twins are individuals having partly different genotypes (they are 50\% correlated for the segregating genes) submitted to similar environmental factors, a stronger similarity of sulcus shape within dizygotic twin pairs when compared to unrelated pairs of individual would also argue that central sulcus similarities may be due to shared genes. As for monozygotic twins, however, similarity may be due to shared genes or shared environment. It thus is the comparison of $\mathrm{G}$ values in monozygotic and dizygotic twins that has to provide the solution.

Values obtained were $G=-1.50$ for the left central sulcus and $G=0.03$ for the right hemisphere, which are not significant at the $\mathrm{P}<0.05$ level. Therefore, we can conclude that the similarity observed for the monozygotic twins is controlled mainly by genetic factors.

\section{Comparison of the Magnitude of T win Resemblance for Sulcal Shape between Monozygotic and Dizygotic Twins}

As said previously the difference in degree of genetic relatedness between monozygotic and dizygotic twins is known, 2:1 (i.e., dizygotic twins are for $50 \%$ correlated for the segregating genes), and this information should be incorporated into a statistical test of heritable influence. To address this issue we used another nonparametric test, the Wilcoxon rank test, to compare magnitude of monozygotic intrapair distances with dizygotic intrapair distances (Norman and Streiner, 1986). Briefly, the procedure consists of doing a rank ordering of all intrapair distances from both samples (monozygotic and dizygotic) combined and doing the summation of the ranks of one group. This value is then used to determine the level of significance. We found $Z=2.03$, meaning that monozygotic intrapair distances are statistically smaller to dizygotic intrapair distances at the $\mathrm{P}<0.05$ level. This test proves that intrapair distances in monozygotic twins are statistically inferior to intrapair distances in dizygotic twins as would be predicted from a genetic model. By proving a significantly higher correlation in monozygotic than in dizygotic pairs, we refute a nongenetic source of the significant intrapair resemblance in monozygotic pairs.

\section{DISCUSSION}

The first step consisted in extracting the median surface of the central sulcus for both hemispheres each individual. We studied under Cortical Sulcus Modeling Using Active Ribbon the accuracy of the Active Ribbon modeling method, demonstrating that this method gives results that are very similar to those obtained from labor-intensive manual delineation. The main advantages of the Active Ribbon method are that (i) it involves a minimum interaction while manual delineation is time consuming and (ii) by stretching a parametric grid over the entire sulcus median surface a structured representation of the median surface of the sulcus is retrieved while manual delineation retrieves an unorganized set of voxels. For intersubject shape comparison purposes, it is particularly important that an anatomical correspondence exists between equivalent points from different surfaces. Figure 3 shows the shape of the mean central sulcus (cf. Eq. (4)) computed from 20 different subjects. The general shape of this "mean" central sulcus exhibits the features commonly found for this structure. In particular the two major genus where the sulcus folds sharply are clearly visible on the mean central sulcus. This argues for the validity of the anatomical correspondence of sulcal surface nodes. While uniform stretching of a parametric grid along a sulcus to construct its parametric representation has also been used by other authors to construct sulcal parameterization (Thompson et al., 1996; Vaillant et al., 1996), another procedure has been introduced recently in Manceaux-Demiau et al. (1998). In this procedure, the major assumption in building a parameterization of a sulcus is that regions in which the sulcus folds sharply can be used as features that determine correspondences across individuals. As noticed by the author, this approach might fail when patterns of crest lines differ considerably. More importantly, in this method the automatic matching of sulcal crest lines is based on the assumption that the 3D locations of these crest lines are stable. Therefore, one would not expect an important difference between a mean sulcus computed from a uniform stretching or a crest-line constrained parameterization because the validity of both relies on spatial correspondence. The subtle shape differences resulting from the utilization of a uniform or constrained parameterization shown by the authors is not prominent enough to change the result of a Principal Component Analysis, which removes, through a modal truncation, high-frequency components of shape differences. Therefore we did not regard crest lines as appropriate landmarks for constraining the parameterization (see also Thompson et al., 1996), for similar discussion).

All parameterized shapes were then resampled in a common referential frame in order to be able to compare the statistics of the coordinates of equivalent points from different shapes in the minimum variance referential. We used a Procrustes fit instead of the Talairach space. In fact, when doing statistical analysis of sulcal variability in Talairach space as in Thomp- 
son et al. (1996), both residual spatial and shape variations of sulcal patterns are mixed. As our study focuses on the analysis of a genetic encoding of a shape, we must remove spatial variability by computing affine transformations before computing statistics on coordinates.

Once modeling using the Active Ribbon method is performed, Principal Component Analysis allows one to obtain, for each sulcal surface $\vec{S}_{k}$, an associated modal vector $\vec{b}_{k}$ representing the most important difference between the given sample and the mean model. By taking only the most important eigenvectors (those associated with the largest eigenvalues) we focus the shape analysis on the main components of its variation. In particular, modal truncation as presented under Statistical Shape Modeling Using PCA allows one to remove high-frequency deformations which are related to both small intersubject differences and segmentation errors.

The existence of genetic influence on the central sulcus shape was addressed by comparing the twin intrapair modal distances with interpair modal distances. Our results highlight a genetic encoding of the central sulcus shape. As previous studies (Bonan et al., 1998) which focused on the comparison of the superficial appearance of the central sulcus (in particular the superficial length) have concluded that nongenetic factors are important for its development, it appears that it is more the deep structure of the central sulcus which is consistent, as several authors have observed (Yousry et al., 1997; White et al., 1997; Lohmann et al., 1999).

Moreover, on monozygotic twins we obtained a $G$ value less significant for the right hemisphere than for the left hemisphere, suggesting that the genetic encoding may be stronger for the left central sulcus compared to the right hemisphere (this was also observed by Tramo et al. (1995) for surface measurements of several gyri on monozygotic twins).

For the simulation of twin pairs, we knew that the shape of the central sulcus was "two times more similar" within a pair of simulated twins $(\sigma=2 \mathrm{~mm}$ for the random number generator) than within other pairs $(\sigma=4 \mathrm{~mm})$. We found values of $\mathrm{G}$ close to -7.0 ( $\mathrm{P}<$ $\left.1.10^{-14}\right)$. When applied to real twins data, $\mathrm{G}$ is significant ( $G=-2.66$; $P<0.005$ for the left hemisphere and $\mathrm{G}=-2.26 ; \mathrm{P}<0.05$ for the right hemisphere) but absolute values are inferior to those obtained in the simulations. Thus, from these simulations, we can say that the magnitude of the difference between twins, $d$, is slightly smaller than the difference observed between two random individuals, $d^{\prime}$, in fact $d^{\prime}>d>d^{\prime} / 2$. Therefore, we argue that nongenetic factors al so play a nonnegligible role in the definition of the central sulcus shape. We conclude, from the observation that a genetic encoding of central sulcus shape is detected in a statistical sense, that the central sulcus shape is more similar between two monozygotic twins than between two dizygotic twins or two random individuals.

\section{CONCLUSION}

Principal Component Analysis can be used to perform sulcal shape comparisons through the computation of modal distances. A statistical test applied on retrieved modal distances (Mantel permutation test) then allows the detection of significant differences between two groups. Using this methodology based on the analysis of, respectively, independent subjects and monozygotic and dizygotic twins, we have shown that a genetic encoding of central sulcus shape is detected.

\section{ACKNOWLEDGMENTS}

The authors express their appreciation for support from the Canadian Medical Research Council (SP-30), the McDonnell-Pew Cognitive Neuroscience Center Program, the U.S. Human Brain Project (HBP), the NIMH, and the NIDA. This work forms part of a continuing project of the HBP-funded International Consortium for Brain Mapping (ICBM) to develop a probabilistic atlas of human neuroanatomy. We also thank The Netherlands Twin Register program for providing us with dizygotic twins data. We thank Keith Worsley and anonymous reviewers for useful comments on statistical analysis.

\section{REFERENCES}

Bailey, P., and Von Bonin, G. 1951. The Isocortex of Man. Univ. of Illinois Pres, Champaign.

Barillot, C., Le Goualher, G., Hellier, P., and Gibaud, B. 1999. Statistical analysis of brain sulci based on active ribbon modeling. In Medical I maging:I mage Processing, Vol. 3661, pp. 312-321. SPIE, Bellingham, WA.

Bonan, I., Argenti, A., Duyme, M., Hasboun, D., Chantome, M., Dormont, D., Marsault, C., and Zouaoui, A. 1998. Abstracts of the 9th International Conference on Twin Studies, Helsinki, Finland: IRM I mages of Central Sulci: A Monozygotic Twins Study. Twin Res. 1:80-117. [Abstract 008]

Bookstein, F. 1989. Principal warps: Thin-plate splines and the decomposition of deformation. IEEE Trans. Pattern Anal. Mach. Intell. 11:567-585.

Bookstein, F. 1991. Morphometric Tools for Landmark Data. Cambridge Univ. Press, Cambridge, UK.

Boomsma, D. I. 1998. Twin registers in Europe: An overview. Twin Res. 1:34-51.

Borgefors, G. 1984. Distance transformation in a arbitrary dimensions. Comput. Vision Graph. I mage Process. 27:321-345.

Caunce, A., and Taylor, C. 1999. Using local geometry to build 3D sulcal models. In IPMI Proceedings, LNCS 1613 (A. Kuba et al., Eds.), pp. 196-209. Springer, Visegrad, Hungary.

Cootes, T., Cooper, D., Taylor, C., and Graham, J . 1992. Trainable method of parametric shape description. Image Vision Comput. 10:289-294.

Crow, E. L., Davis, F. A., and Maxfield, M. W. 1990. Statistical Manual. Dover, New York.

Csernansky, J ., J oshi, S., Wang, L., Haler, J ., Gado, M., Miller, J ., Grenander, U., and Miller, M. 1998. Hippocampal morphometry in schizophrenia by high dimensional brain mapping. Proc. Natl. Acad. Sci. USA 95:11406-11411. 
Dumoulin, S., Bittar, R., Kabani, N., Baker, C., J r., Le Goualher, G., and Evans, A. 1998. Quantification of the variability of human area $\mathrm{V} 5 / \mathrm{MT}$ in relation to the sulcal pattern in the parieto-temporo-occipital cortex: A new anatomical landmark. Neuroimage 7(4):S319.

Kamada, K., Oshiro, O., Takeuchi, F., Kuriki, S., Houkin, K., I wasaki, Y., and Abe, H. 1993. Identification of central sulcus by using somatosensory evoked magnetic fields and brain surface MR images: Three dimensional projection analysis. J. Neurol. Sci. 116:29-33.

Le Goualher, G., Barillot, C., and Bizais, Y. 1997. Modeling cortical sulci using active ribbons. Int. J. Pattern Recognit. Artif. Intell. 11:1295-1315.

Le Goualher, G., Barillot, C., Le Briquer, L., and Bizais, Y. 1995. 3D detection and representation of cortical sulci. In Computer Assisted Radiology (H. U. Lemke, K. Inamura, C. C. J affe, and R. Felix, Eds.), pp. 234-240. Springer-Verlag, Berlin.

Le Goualher, G., Procyk, E., Collins, L., Venegopal, R., Barillot, C., and Evans, A. 1999. Automated extraction and variability analysis of sulcal neuroanatomy. IEEE Trans. Med. Imag. 18:206-216.

Lohmann, G., von Cramon, D., and Steinmetz, H. 1999. Sulcal variability of twins. Cerebr. Cortex 9:754-763.

Manceaux-Demiau, A., Nick Bryan, R., and Davatzikos, C. 1998. A probabilistic ribbon model for shape analysis of the cerebral sulci: Application to the central sulcus. J. Comput. Assisted Tomogr. 22:962-971.

Mantel, N. 1967. The detection of disease clustering and a generalized regression appraoch. Cancer Res. 27:209-220.

Martin, J., Pentland, A., Sclaroff, S., and Kikinis, R. 1995. Characterization of neuropathological shape deformations. IEEE Trans. Pattern Anal. Mach. Intell. 17:545-561.

Mazziota, J., Toga, A., Evans, A., Fox, P., and Lancaster, J . 1995. A probabilistic atlas of the human brain: Theory and rationale for its development. Neurol mage 2:89-101.

Mortenson, M. 1985. Geometric Modeling. Wiley, New York.

Norman, G., and Streiner, D. 1986. PDQ Statistics. B. C. Decker, Hamilton, Ontario.

Ono, M., Kubick, S., and Albernathey, C. 1990. Atlas of the Cerebral Sulci. Thieme, Stuttgart.

Oppenheim, J ., Skerry, J ., and Tramo, M. 1979. Magnetic resonance imaging morphology of the corpus callosum in monozygotic twins. Ann. Neurol. 26:100-104.

Sclaroff, S., and Pentland, A. P. 1995. Modal matching for correspondence and recognition. IEEE Trans. Pattern Anal. Mach. Intell. 17:545-561.
Shapiro, C., and Hubert, L. 1979. Asymptotic normality of permutation statistics derived from weighted sums of bivariate functions. Ann. Stat. 7:788-794.

Sibson, R. 1978. Studies in the robustness of multidimensional scaling: Procrustes statistics. J . R. Stat. Soc. 40:234-238.

Sorlié, C., Collins, D., Worsley, K., and Evans, A. 1997. An anatomical variability study based on landmarks. McConnell Brain Imaging Center, Montreal Neurological Institute, McGill University, Montreal. [Technical report]

Steinmetz, H., Herzog, A., Huang, Y., and Hacklande, T. 1994. Discordant brain-surface anatomy in monozygotic twin. N. Engl. J. Med. 331:952-953.

Steinmetz, H., Herzog, A., and Schlaug, G. 1995. (A)symmetry in monozygotic twins. Cereb. Cortex 5:296-300.

Thompson, P., Schwartz, C., Lin, R. T., Khan, A. A., and Toga, A. W. 1996. Three-dimensional statistical analysis of sulcal variability in the human brain. J . Neurosci. 16:4261- 4274.

Tramo, J ., Loftus, W., and Thomas, C. 1995. Surface area of human cerebral cortex and its gross morphological subdivisions: In vivo measurements in monozygotic twins suggest differential hemisphere effects of genetic factors. J . Cognit. Neurosci. 7:292-301.

Vaillant, M., Davatzikos, C., and Bryan, R. 1996. Finding 3D parametric representations of deep cortical folds. In Workshops on Math: Methods in Biomedical Image Analysis, pp. 151-159.

Watson, J ., Myers, R., Frackowiak, R., Hajnal, J ., Woods, R., Mazziota, J., Shipp, S., and Zeki, S. 1993. Area V5 of the human brain: Evidence from a combined study using positron emission tomography and magnetic resonance imaging. Cereb. Cortex 3:79-94.

Weinberger, D., Bartley, A., and J ones, D. 1992. Regional cortical gyral variations in human monozygotic twins. Soc. Neurosci. Abstr. 12:595.

White, L., Andrews, T., Hulette, C., Richards, A., Groelle, M., Paydarfar, J ., and Purves, D. 1997. Structure of the human sensorimotor system. I. Morphology and cytoarchitecture of the central sulcus. Cereb. Cortex 7:18-30.

Worsley, K., Evans, A., Strother, S., and Tyler, J. 1991. A linear spatial correlation model, with applications to positron emission tomography. J . Am. Stat. Assoc. 86:55- 67.

Yousry, T., Schmid, U., Alkadhi, H., Schmidt, D., Peraud, A., Buettner, A., and Winkler, P. 1997. Localization of the motor hand area to a knob on the precentral gyrus: A new landmark. Brain 120:141-157.

Zilles, K., Schleicher, A., Langemann, C., et al. 1997. Quantitative analysis of sulci in the human cerebral cortex: Development, region heterogeneity, gender difference, asymmetry, inter-subject variability and cortical architecture. Hum. Brain Mapp. 5:218-221. 